Ces deux échantillons $1^{\circ}$ et $2^{\circ}$ accusaient respectivement après 24 heures de séjour à l'étuve :

$1^{0}$ Le lait non essoré : $34^{\circ}, 6$.

2 . Le même lait essoré : $35^{\circ}, 2$.

Essai du 19 juin 1924 :

10 Lait eru avant 1'essorage : 70,4.

$2^{\circ}$ Même lait après essorage : $7^{\circ}, 0$.

Après 8 heures de séjour à l'étuve respectivement :

10 Lait avant essorage : $17^{\circ}$.

$2^{\circ}$ Lait après essorage : $17^{\circ}, 8$.

On ne peut done douter que se produit, ou, pour être plus précis, que s'amorce au cours de l'essorage, une légère augmentation du nombre des mierobes véhiculés par le lait cru. Cette plus forte poussée microbienne, portant naturellement sur les ferments lactiques, doit se rattacher, très vraisemblablement, à la très sensible aératìon, accompagnée de fixation d'oxygène, que le lait subit dans cette opération, et qui est présisément l'un des inconvénients de l'essorage centrifuge dont nous venons de parler.

\title{
LA VITAMINE C DANS LE LAIT CONCENTRÉ SUCRÉ
}

\section{par Dr A. BAKKE}

Chef des Services Scientifiques de la Nestlé \& Anglo-Swiss Condensed Milk Co.,

Assisté par

Mile V. ASCHEHOUG,

Assistante au Laboratoire Central de la N. \& A. S. C. M. Co à Vevey.

\section{(Suite et fin)}

Nous avons démontré dans un précédent article que la vitamine $C$ du lait frais résiste parfaitement au eours de la fabrieation du lait con zentré sucré, lorsque celle-ci se fait dans de bonnes conditions.

En outre, nous avons établi que les propriétés antiscorbutiques du lait frais se conservent longtemps dans le lait concentré sucré.

Il était intéressant de vérifier que la vitamine $\mathrm{C}$ du lait concentré sucré reste active pendant une période plus longue encore que la période envisagée lors des premiers essais. Des travaux ont été faits en France dans cet ordre d'idées il y a quelques années, mais les relations qui en ont été publiées sont trop peu détaillées pour qu'il soit possible de suivre de près la marche des observations. Il va sans dire qu'il est plus facile d'obtenir des précisions de ce genre dans notre laboratoire, où l'on utilise et examine les produits de toutes les fabriques Nestlé que notre Service scientifique a pour mission de eonseiller.

Lors de nos premières expériences, nous avions réservé, afin de l'examiner plus tard, une partie du lait concentré sucré utilisé, de sorte que la nouvelle série d'expériences dont nous allons dire quelques mots, 
porte sur du lait de même fabrication que dans nos séries précédentes, mais déjà vieux de 8 à 10 mois $1 / 2$.

Nos nouveaux essais ont prouvéque les propriétés antiscorbutiques de ce lait eonzentré déjà vieux n'a vaient pas changédurant ce la ps de temps.

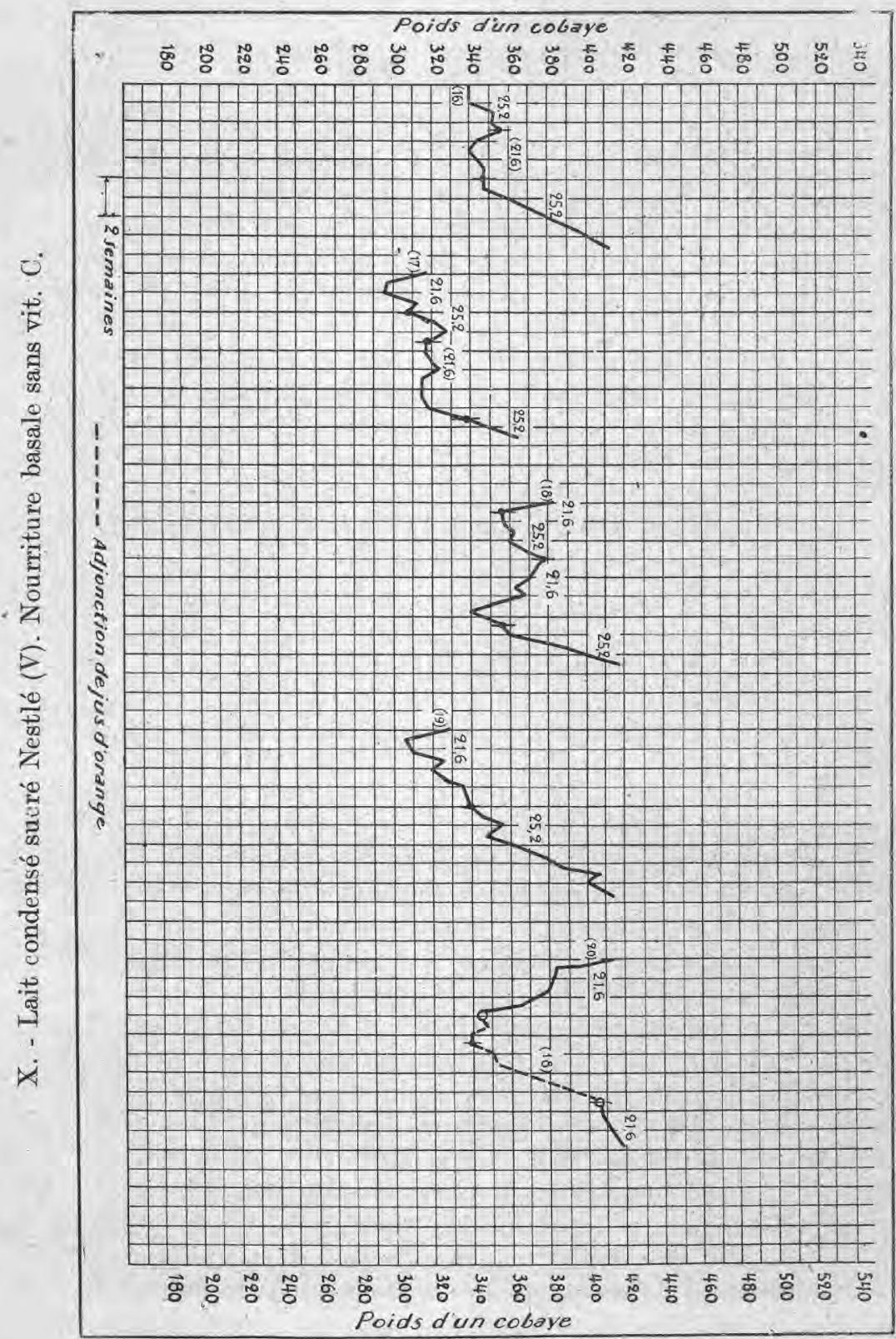


Las gra phiques ci-dessous illustrent les résultats de ces nouveaux essais, quí corroborent ce que nous avions a vancé dans notre premier article. Voyons d'abord les graphiques X et XI. Ils correspondent aux gra-

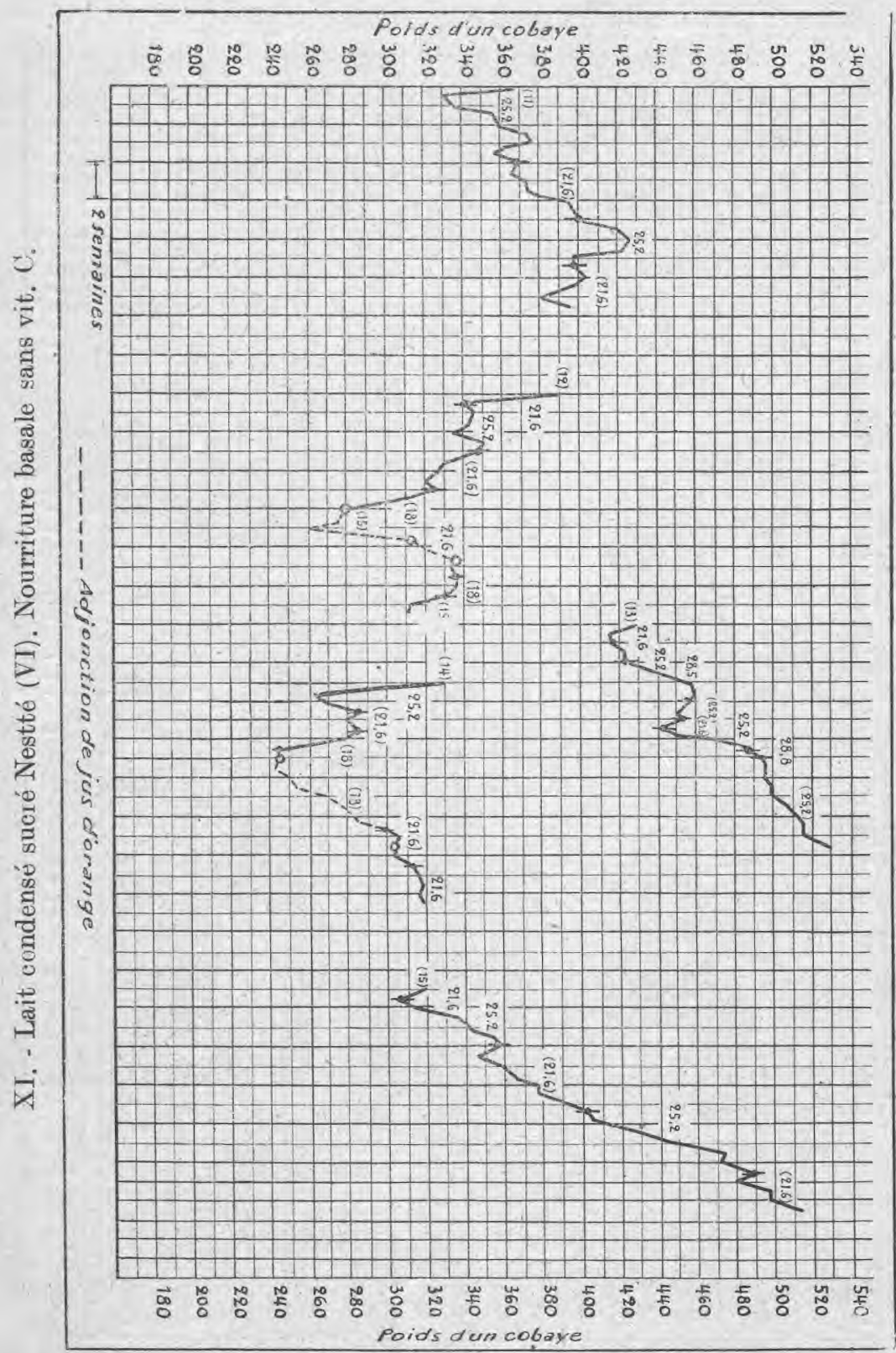


phiques V et VI de la première série d'essais. Nous a vons indiqué entre parenthèses la quantité de lait concentré réellement absorbée par les cobayes. Nous sommes arrivés à ees chiffres en pesant la nourriture refusée et en la déduisant de la ration prévue. (Le premier chiffre entre parenthèses représente le numéro de l'animal.)

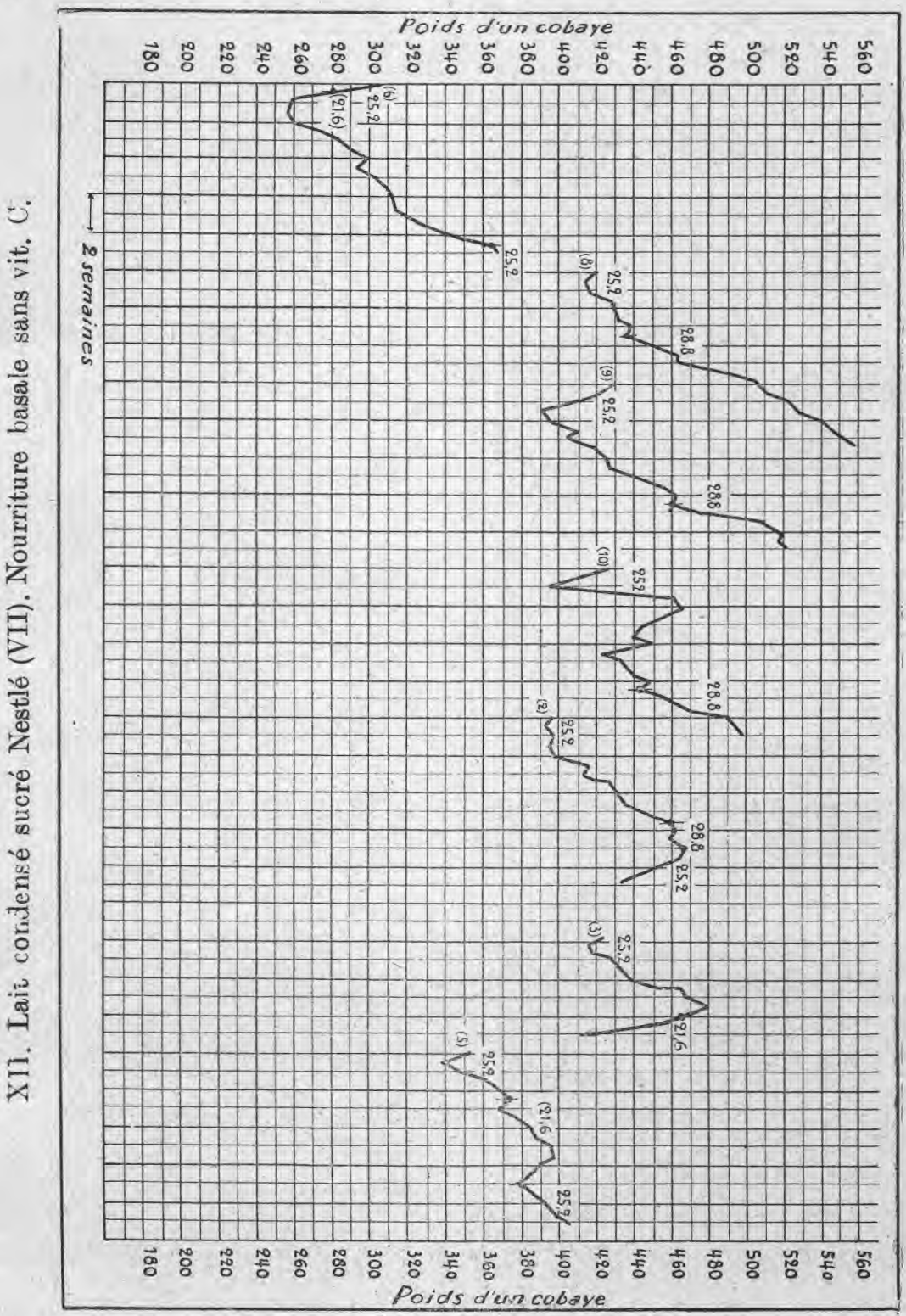


La comparaison de ces deux graphiques, résumant les séries obtenues avec du lait concentré d'âge divers, nous permet de constater, une fois de plus, que les cobayes sont, en général, réfractaires au lait concentré. Le développement de quelques sujets n'a cependant pas souffert, la quantité de lait ingérée étant sans doute restée suffisante. D'autres ont repris après adjonction de jus d'orange à leur régime, et se sont développés régulièrement à partir de ce moment. Au contraire, certains animaux ont de nouveau dépéri dès qu'on a cessé de leur donner du jus d’orange.

Nous voyons aussi que des quantités de lait sensiblement identiques ont suffi dans les deux séries d'expériences. Les cobayes nourris avec le second lot de lait concentré ont reçu une dose initiale un peu plus forte, mais ensuite, des rations plutôt inférieures à celles des premiers essais ont suffi à leur assurer un développement régulier.

Nous avons déjà signalé les difficuilté auxquelles on se heurte en voulant nourrir des cobayes au lait concentré. Compte tenu de cet important facteur, il appert des gra phiques que l'activité antiscorbutique est rèstée sensiblement la même dans le lot de lait plus âgé.

Passant au graphique XII qui correspond au graphique VII de notre premier article, on voit que les résultats de ces deux séries d'essais, portant sur des laits d'âge divers, sont presque identiques.

Malheureusement, les sujets dont les graphiques XIII et XIV (correspondant aux graphiques VHI et IX de notre premier article) montrent le développement, étaient extrêmement réfractaires au lait concentré sucré. Il n'en ressort pas moins que cet aliment suffit à couvrir les besoins en vitamine $\mathrm{C}$ d'un cobaye, puisque les animaux qui ont ingéré du lait, et cela même en quantités modérées, accusent une courḅe satisfaisante.

Ces tableaux, et c'est là leur intérêt particulier, attestent donc de façon frappante que, si parfois les cobayes ne prospèrent pas au lait concentré, on le doit à ce qu'ils ont refusé une partie de leur ration quotidienne.

Faute d'une réserve suffisante en lait fabriqué l'été précédent, nous avons dû interrompre cette seconde série d'essais plus tôt que la première.

Ce qui précède démontre suffisamment que J'activité antiscorbutique ne s'est pas atténuée dans le lait concentré sucré entre la première et la seconde série de nos essais. On enregistre d'abord une légère diminution - sans grande portée d'ailleurs - de cette activité, aussitôt après la fabrication, puis elle semble ensuite rester constante pendant environ une année.

Or, dans la pratique, du lait concentré sucré de bonne marque, assuré d'un débit régulier, est consommé bien a vant d'avoir atteint-cet âge.

Inutile d'ajouter que le lait utilisé pour nos expériences a été prélevé sur des fabrications courantes et conservé dans des conditions identiques à celles du lait concentré mis dans le commerce, c'est-à-dire simplement en boîtes stérilisées a vant remplissage et étanches à l'air. 







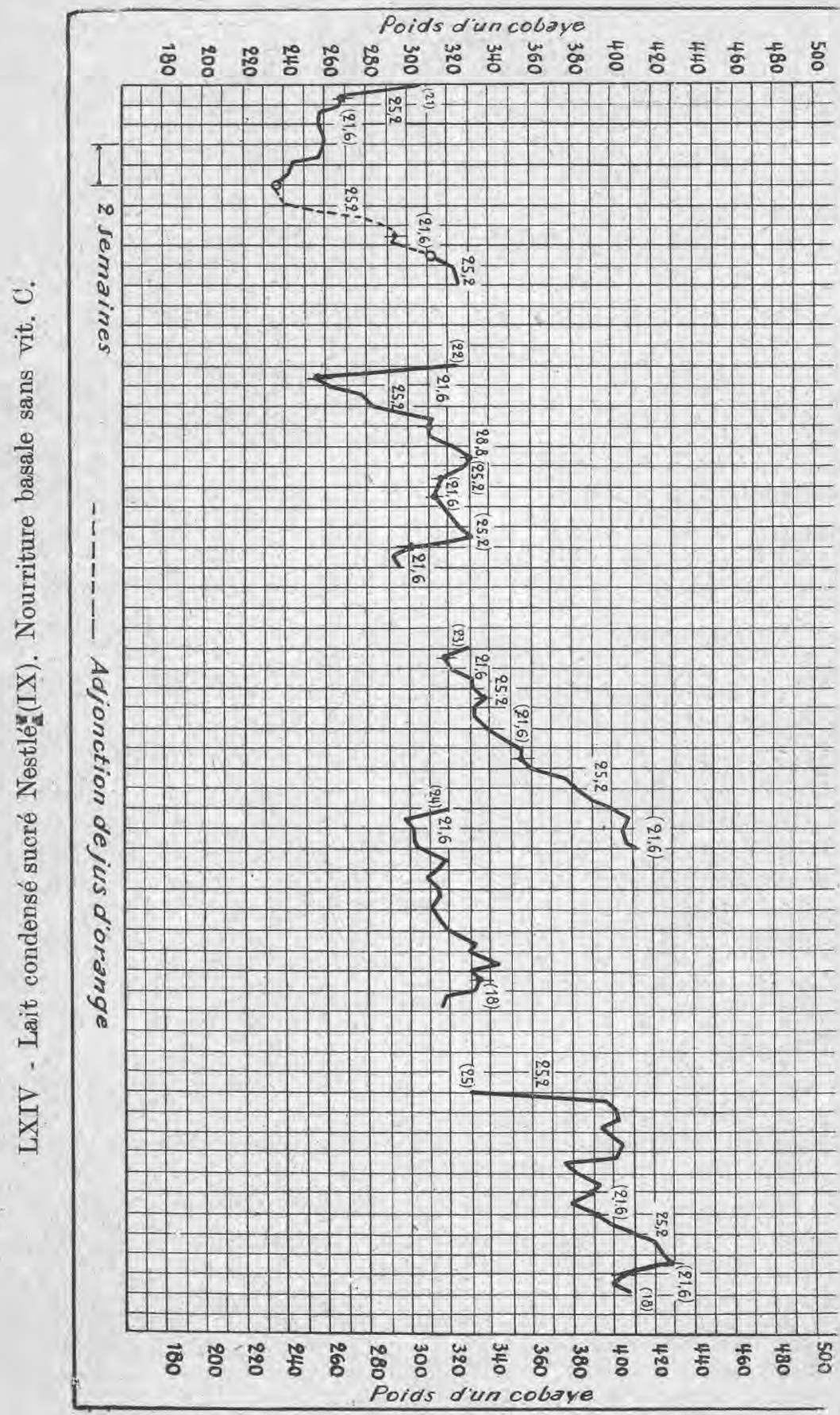

